Curr Opin Chem Biol. 2008 December ; 12(6): 612-618. doi:10.1016/j.cbpa.2008.09.033.

\title{
RNA in MOTION
}

\author{
Kathleen B. Hall \\ Dept of Biochemistry and Molecular Biophysics, Washington University School of Medicine, St \\ Louis, MO63110 kathleenhal@gmail.com
}

\section{Summary}

Although RNA duplex regions are highly structured and inflexible, other elements of an RNA molecule are capable of dynamic motions. These flexible regions are sites of interactions with small molecules, proteins, and other RNAs, yet there are few descriptions of these regions that include the timescale and amplitude of their motions. No one technique is sufficient to accurately describe these motions, but the combination of in vitro methods, particularly NMR relaxation methods, and more robust in silico methods, is beginning to yield the type of data that can be used to understand RNA function. Very few RNAs have been described by both techniques, and here one such RNA and one RNA:protein complex are reviewed.

\section{Introduction}

Why is it not enough to simply say that an RNA is floppy? In the case of a floppy RNA hairpin loop, its disorder could be important for adaptation to a protein binding site or an RNA receptor site. Unstructured RNA sequences are binding sites for proteins; internal bulges in an RNA duplex distinguish an miRNA regulatory site from an RNAi cleavage site; tRNA anticodons base pair with mRNA codons. In the same way that the hub hypothesis of intrinsically disordered proteins (1) describes how a single protein sequence can bind different ligands, an unstructured RNA has the same potential. While this review centers upon the most current efforts to characterize RNA dynamics, it must be noted that the functional significance of the motions remains to be determined. Figure 1 shows those RNAs for which there are both in vitro and in silico data.

\section{The smallest RNA}

This ubiquitous ${ }^{5}$ UUCG tetraloop RNA hairpin is found in large RNAs, where it is thought to act as a nucleation site for global folding by controlling the folding pathway. There are no known natural ligands for the UUCG tetraloop, and it has not been found in RNA:RNA tertiary interactions. There are many excellent studies of this hairpin, but here I consider only recent investigations using the combination of NMR and Molecular Dynamics simulation.

\section{NMR dynamics}

The $\mathrm{U}_{1} \mathrm{U}_{2} \mathrm{C}_{3} \mathrm{G}_{4}$ tetraloop is an intricately connected structure (Figure 2). Ferner et al., (2) looked at the dynamics of the structure as a function of temperature, essentially probing the unfolding pathway. Their NMR experiments used ${ }^{13} \mathrm{C} /{ }^{1} \mathrm{H}$ as reporters of dynamics where they monitored the $\mathrm{C} 6 \mathrm{H} 6 / \mathrm{C} 8 \mathrm{H} 8$ spin systems of the bases and the $\mathrm{C} 1^{\prime} / \mathrm{H} 1^{\prime}$ of the riboses. NMR

\footnotetext{
Publisher's Disclaimer: This is a PDF file of an unedited manuscript that has been accepted for publication. As a service to our customers we are providing this early version of the manuscript. The manuscript will undergo copyediting, typesetting, and review of the resulting proof before it is published in its final citable form. Please note that during the production process errors may be discovered which could affect the content, and all legal disclaimers that apply to the journal pertain.
} 
relaxation data were analyzed using the Lipari-Szabo formalism $(3,4)$, which extracts an order parameter $\left(S^{2}\right)$ and bond vector correlation times for each probed site. The order parameter is a measure of the rapid (ps-ns) spatial variation of the vector position within the molecular framework. Values range from $0 \leq S^{2} \leq 1$, where $S^{2}=1$ indicates no local motion and $S^{2}=0$ indicates isotropic local motion. The same parameters can be calculated from MD simulations, providing a direct comparison of the two experiments.

NMR experiments on the UUCG tetraloop were conducted at three temperatures: $298 \mathrm{~K}, 317$ $\mathrm{K}$, and $325 \mathrm{~K}(2)$. These temperatures were selected for the comparison of the UUCG tetraloop internal dynamics with those of the structurally related uCACGg tetraloop. The highest temperature used is in the pre-melting region of the uCACGg hairpin. Although the $\mathrm{U}_{1} \mathrm{U}_{2} \mathrm{C}_{3} \mathrm{G}_{4}$ RNA is unusually stable, its structure is not static. As shown in Figure 2, $\mathrm{U}_{2}$ extends out into solution, and NMR relaxation data show that the base undergoes significant mobility $\left(S^{2}=\sim 0.7\right)$. The motion is about the glycosidic bond $(\chi)$, and does not include ribose dynamics as shown by a nearly uniformly high order parameter for riboses in the loop and the stem. As the temperature increases to $317 \mathrm{~K}$, there is little change in the pattern of dynamics: $\mathrm{U}_{2}$ becomes more dynamic $\left(S^{2}=0.6\right)$ and the adjacent $C_{3}$ appears to move more rapidly. Some fluctuations in the riboses of the loop become visible.

\section{Simulation Dynamics}

Starting structure for the UUCG tetraloop came from a previous simulation, while the uCACGg structure was taken from 1RFR.pdb (2). MD experiments used GROMACS with the AMBER parm98 force field; simulations were run in NPT mode, using PME for long range electrostatics, for $50 \mathrm{~ns}$. Order parameters calculated from MD trajectories are in excellent agreement with the NMR order parameters, although noting that the order parameters calculated from the simulation are consistently lower (i.e. more motion) than those from the NMR experiments.

While the UUCG tetraloop is a model system in many ways, its stable structure can obscure any discrepancies between in vitro and in silico experiments that relate to the description of very dynamic systems. Thus the comparison of the two experiments with the $\mathrm{uC}_{1} \mathrm{~A}_{2} \mathrm{C}_{3} \mathrm{G}_{4} \mathrm{~g}$ hairpin is most illuminating, since this hairpin melts near $325 \mathrm{~K}$. At $298 \mathrm{~K}$, the NMR data show that the $\mathrm{A}_{2}$ base is very floppy $\left(\mathrm{S}^{2}=0.6\right)$. Ribose dynamics are also more apparent in this RNA, although the authors caution that the NMR data were difficult to fit. Simulation data reproduce the NMR base dynamics quite well, but the ribose dynamics are quite uniform in the MD, without the wiggles seen in the NMR data. As the temperature increases to the pre-melting value, the agreement between MD and NMR data is reduced, but both show base motions now throughout the loop and fraying of the stem.

To analyze the MD trajectories, the authors used principal component analysis to uncover dynamic networks. These analyses allowed them to discern distinct populations of structures (clusters) that in solution would be averaged and difficult to distinguish. Their data suggested that there were two conformational states of the hairpins at lower temperatures, with transitions occurring on the order of $10 \mathrm{~ns}$. Clusters were also present at the highest temperature measured, rather than a continuum of dynamic structures. This result agrees with their replica exchange MD (5) and also with temperature-jump folding experiments (6), which revealed several conformational states of the RNA.

A folding experiment with the minimal 5'gcUUCGgc hairpin used Replica Exchange MD, which is a method to sample the conformations of a molecule through temperature jumps. The experiment began with an extended unstacked RNA chain (7). All 52 replicas folded to the canonical UUCG structure at least once during the simulation, but there were ensembles of 
structures that deviated from normal. Of particular interest is the cluster of structures that differ in base stacking and hydrogen bonding in the loop.

These new in vitro and in silico experiments suggest that the UUCG tetraloop is not a static structure, but samples conformational substates. MD data in particular show multiple conformations, and if their energetics are comparable to the canonical structure, then they could be populated in solution. If those structures are present, then we should find them; if the conformational heterogeneity is an artifact of the simulation conditions, then the cause needs to be identified and fixed.

\section{The Largest RNA}

Dynamic processes are at the heart of the ribosome enzyme [view the movies in Protein Factory Reveals Its Secrets C\&E News (2007) 85(8)13-16] but only the L11 protein bound to the GTPase-associated center (GAC) is described here. RNA elements within this center include the two helices $\mathrm{H} 43$ and $\mathrm{H} 44$ where protein L11 binds (Figure 1). L11 has two domains that are structurally distinct: the C-terminal domain (CTD) binds the RNA, and the N-terminal domain (NTD) might bind to cofactors (Figure 3). L11 NTD appears in various orientations in different crystal structures of the ribosome, as quantified by Ilin et al. (8). Several NMR studies have explored the structure and dynamics of the complex formed by L11 and RNA $(9,10)$, and also came to different conclusions about the relative orientation of L11 on the RNA.

\section{L11 dynamics by NMR}

The CTD of L11 binds the GAC site through interactions of the flexible recognition loop connecting $\alpha 1$ and $\beta 1$ (Figure 3). NMR ${ }^{15} \mathrm{~N}$ relaxation data $\left(\mathrm{T}_{1}, \mathrm{~T}_{2}\right.$, and $\mathrm{NOE}$ ) reporting on the rapid dynamics of the backbone amides show that this loop is very mobile on the picosecond/ nanosecond timescale in the free $\mathrm{L} 11$ proteins from Bacillus stearothermophilus at $25^{\circ} \mathrm{C}$ (11), Thermus thermophilus at $40^{\circ} \mathrm{C}(9)$ and Haloarcula marismortui at $27^{\circ} \mathrm{C}(10)$.

In the free L11 proteins from T. thermophilus and H. marismortui, the NTD appears to have more intrinsic flexibility than does the core of the CTD. $\mathrm{T}_{1 \rho}$ and $\mathrm{T}_{2}$ values measured in ${ }^{15} \mathrm{~N}$ NMR relaxation experiments are longer for the NTD, while its ${ }^{15} \mathrm{~N} /{ }^{1} \mathrm{H}$ heteronuclear NOEs are lower. Both measurements are consistent with rapid backbone motions. In the $T$.

thermophilus protein, a single amino acid shows anomalous dynamics: Alanine 51 is at the end of the second $\beta$-strand of the NTD, and its backbone amide clearly displays rapid motion (9). The flanking residues are somewhat less mobile. In addition to rapid motions near Ala51, there were also slower motions occurring around this site, specifically at the adjacent Gly49. This region of the protein appears to be a hinge, connecting the $\alpha$-helix with the sheet. The other site showing slower ( $\mu$ s-ms) dynamics is Ser75, which flanks the linker region. At least one flanking residue had unusual dynamic properties on the fast timescale for the $H$. marismortui protein, while the region clearly had more rapid motions in the T. thermophilus protein.

The picture of the protein backbone dynamics from NMR is that of locally fast fluctuations of the unstructured RNA recognition loop and some internal dynamics of the NTD, but no large amplitude inter-domain motions. However, in the complex with GAC RNA, the L11 protein domains change their relative orientations (Figure 4) and their local dynamics. Differences in the $\mathrm{L} 11$ free and bound ${ }^{15} \mathrm{~N}$-relaxation parameters for the $\mathrm{H}$. marismortui complex are most dramatic, for the flexibility in the long RNA recognition loop completely disappears when RNA is bound. The calculated order parameters of the backbone amide indicate a uniformly damped motion of both C- and N-terminal domains (10). For the T. thermophilus complex (9), there remains residual motion of the RNA recognition loop as well as of the backbone amides around and including Ala51, although the motions are damped. Since the NTD does 
not interact with RNA, changes in its dynamics upon RNA binding suggest some coupling/ correlation that remains to be identified.

The NTD orientation is not well defined. In the T. thermophilus protein:RNA complex, the position of the NTD appears to have rotated by about $70^{\circ}$ around the long axis of the complex. The authors trace the movement to torsion angles in both domains and within the linker region (9) through a comparison of the NMR structure with cryo-EM and crystal structures. Those amino acids that exhibited unusual backbone dynamics (ps-ns and $\mu \mathrm{s}-\mathrm{ms}$ ) were also those that appeared to be responsible for the change in domain orientation. This correlation suggested that intrinsic motions of the free protein allow the global deformation of the protein in the complex to happen with little free energy penalty. In the H. marismortui complex, NMR data (10) show that the two domains of L11 have different overall tumbling times, suggesting that the NTD has more relative movement in the complex.

\section{Molecular dynamics simulations}

Simulation data of the T. maritima L11:GAC complex (12) can be compared to the L11 NMR relaxation data and also provides new data on the GAC. The starting structure was the x-ray crystal structure (1MMS; (13)), where the NTD does not contact the RNA, and has a higher B-factor than the rest of the complex. Three $16 \mathrm{~ns}$ simulations included the 58 nucleotide RNA GAC, L11, TIP3P waters and $\sim 0.1 \mathrm{M} \mathrm{NaCl}$. MD runs used the Sander module of AMBER7 and the Cornell force field with PME.

The general result from these simulations was that the CTD and the RNA did not deviate much from the starting crystal structure, while the NTD showed significant fluctuations.

Conformational changes observed in all simulations were 1) the NTD underwent rotations about the linker to become more or less packed against the RNA; 2) RNA nucleotide A1067 ( $E$ coli numbering) flipped in and out of the RNA structure to be either protected or exposed to solvent.

Within L11, only the NTD showed significant fluctuations within its structure. It appears that most regions of the domain exhibited rapid motions, as reported by the fluctuations of the $\mathrm{C} \alpha$ position. A conformational change of nearly $2 \AA$ was centered in the domain adjacent to the linker, and other local regions of the domain are dynamic. Perhaps these fluctuations are unique to a given protein, since they do not appear in the NMR data of the H. marismortui protein, but are present in L11 from T. thermophilus.

Fluctuations of the T. maritima GAC RNA in the complex were located around the A1067 loop (Figure 5), as monitored by the phosphate fluctuations at A1067/G1068 and A1073. The A1067 $\chi$ torsion angle underwent syn/anti/-syn flips; notably, this appeared to be a very local conformational change. Other sites of RNA flexibility were the A1095 in the second RNA loop and the junctions between stems. These junctions are potentially hinge regions, but in these simulation, no bending motions were observed (their timescale would be too long to be detected).

A $30.5 \mathrm{~ns}$ MD simulation of the H. marismortui GAC alone was carried out to examine the hinge motions in a larger construct (14). The starting structure was from the $50 \mathrm{~S}$ crystal (1JJ2; (15)) and the simulation box contained TIP3P waters, only neutralizing $\mathrm{Na}^{+}$, with PME. Motions in the GAC helix 43/44 region are described as internal breathing, attributed to the conformational fluctuations of the non-canonical base pairs. As in the simulation of the $T$. maritima complex, the bases of the A1067 (A1171 in Hm) loop at the end of H43 exhibit conformational flexibility, with A1171 undergoing a syn/anti transition. One base triple (G1175 in Hm, G1074 in Ec) that forms with a C:G base pair in H44 is dynamic (C:G/G), and $\mathrm{Na}^{+}$ions exchange with bridging water molecules at the $\mathrm{G} / \mathrm{G}$ interface. Bridging water 
molecules in non-Watson-Crick base interactions appear often in the junction between $\mathrm{H} 43$ and H44. Species-specific conformational dynamics of the GAC could be functionally significant, since in vivo environments differ in their salt concentration, specific ion types, and ambient temperature. The challenge is to devise experiments to determine which conformational dynamics of the GAC (or any RNA) are fundamental to the biology, and which are simply the natural fluctuations that all molecules undergo.

\section{The ribosome}

L11 and the GAC are part of the ribosome, of course. The behavior of the L11:GAC complex was observed in all-atom simulations of the 70S ribosome (16) using targeted MD. The complex can be seen interacting with the incoming tRNA during the initial encounter stage. In particular, A1067 makes progressive contact first with tRNA bases, then with the tRNA backbone during this time, as if guiding it into place. This conformation of A1067 corresponds to the flipped-out state seen in the simulation of the L11/GAC complex. The position of the $\mathrm{N}$-terminal domain of L11 is not clear in the trajectory, but the prediction from these studies of the isolated complex is that it should swing into place during the initial encounter stage.

\section{RNA Dynamics to come}

What needs to be done to enhance the synergy between in vitro and in silico experiments? Certainly a fundamental issue is salt. RNAs require cations to neutralize their phosphates, but those cations require anions to satisfy thermodynamic balance. The salt concentration determines the melting temperature of nucleic acids, and as the length of simulations approaches the timescale of RNA folding, the salt will matter. The continuing evolution of force fields and parameters for nucleic acids will be greatly facilitated by comparison with NMR relaxation data (17). Many RNAs use $\mathrm{Mg}^{2+}$ counterions to facilitate folding, and the theoretical problem of capturing the properties of this highly polarizable ion is not yet solved (18). Conformational changes can be monitored on the ps-ns- $\mu$ s timescale by NMR for comparison to simulation.

There are no in vitro dynamics data for the GAC RNA, and so the computational data cannot be compared to solution studies. The GAC RNA structure is intricate and NMR studies of its structure are hampered by its noncanonical interactions and the contacts of phosphates with ions that are not easily observed with NMR methods. This situation, alas, is likely to be normal for biologically interesting RNAs, and points out the desperate need for robust computational methods to examine their properties. Where a direct comparison of conformational dynamics is impossible, then simulations could be used as a guide for nucleotide substitutions that selectively alter the RNA motions, and these mutated RNAs are then examined in vitro (by NMR, fluorescence, or fast folding experiments) as well as in vivo to compare function. Successful studies of RNA structure, dynamics, and function must utilize in vitro, in silico, and in vivo methods to characterize these active molecules.

\section{Acknowledgements}

First, I must profusely apologize to the many people whose excellent work I have not described or cited. This work was supported in part by the National Institutes of Health (R01-GM046318).

\section{References}

1. Dosztanyi Z, Chen J, Dunker AK, Simon I, Tompa P. Disorder and sequence repeats in hub proteins and their implications for network evolution. J Proteosome Res 2006;5:2985-2995.

$\bullet$ 2. Ferner J, Villa A, Duchardt E, Widjajakusuma E, Wohnert J, Stock G, Schwalbe H. NMR and MD studies of the temperature-dependent dynamics of RNA YNMG-tetraloops. Nucleic Acids Res 
2008;36:1928-1940. [PubMed: 18272534]The authors use the Al-Hashimi technique of lengthening the stem of the RNA to accurately measure ${ }^{13} \mathrm{C}$-NMR relaxation parameters of the UUCG and CACG tetraloops. The agreement between in vitro and in silico is rather good. NMR spectra were acquired in $10 \mathrm{mM}$ potassium phosphate, $\mathrm{pH} 6.2,40 \mathrm{mM} \mathrm{KCl}, 0.2 \mathrm{mM}$ EDTA. MD simulations used TIP3P waters but only 13 sodium ions for neutralization.

3. Lipari G, Szabo A. Model-free approach to the interpretation of nuclear magnetic resonance relaxation in macromolecules. 1 Theory and range of validity. J Amer Chem Soc 1982;104:4546-4559.

4. Lipari G, Szabo A. Model-free approach to the interpretation of nuclear magnetic resonance relaxation in macromolecules. 2 Analysis of experimental results. J Amer Chem Soc 1982;104:4559-4570.

-5. Villa A, Widjajakusuma E, Stock G. Molecular dynamics simulation of the structure, dynamics, and thermostability of the RNA hairpins uCACGg and cUUCGg. J Phys Chem B 2008;112:134-42. [PubMed: 18069816]Replica-exchange MD in TIP3P and TIP4P waters. Only neutralizing $\mathrm{Na}^{+}$ counterions. The UUCG loop was more flexible than the CACG, even though there are more hydrogen bonding interactions in the UUCG loop.

6. Ma H, Proctor DJ, Kierzek E, Kierzek R, Bevilacqua PC, Gruebele M. Exploring the energy landscape of a small RNA hairpin. J Am Chem Soc 2008;128:1523-1530. [PubMed: 16448122]

-7. Garcia AE, Paschek D. Simulation of the Pressure and Temperature Folding/Unfolding Equilibrium of a Small RNA Hairpin. J Am Chem Soc 2008;130:815-817. [PubMed: 18154332]The minimal UUCG hairpin in studied by Replica Exchange MD in a box of TIP3P waters, effectively $150 \mathrm{mM}$ $\mathrm{NaCl}$, using Amber ff99 in GROMACS, with $270 \leq \mathrm{T} \leq 601 \mathrm{~K}$. See the folding movie in supplementary material.

8. Ilin S, Hoskins A, Ohlenschlager O, Jonker HRA, Schwalbe H, Wohnert J. Domain reorientation and induced fit upon RNA binding: solution structure and dynamics of RNA protein L11 from Thermotogo maritima. ChemBioChem 2005;6:1611-1618. [PubMed: 16094695]

••9. Lee D, Walsh JD, Yu P, Markus MA, Choli-Papadopoulou T, Schwieters CD, Krueger S, Draper DE, Wang YX. The structure of free L11 and functional dynamics of L11 in free, L11-rRNA(58 nt) binary and L11-rRNA(58 nt)-thiostrepton ternary complexes. J Mol Biol 2007;367:1007-1022. [PubMed: 17292917]The L11 protein from Thermus thermophilus is studied here by $\mathrm{NMR}^{15} \mathrm{~N}$ relaxation to measure rapid (ps-ns) fluctuations. Solutions contained $50 \mathrm{mM} \mathrm{NaCl}, 20 \mathrm{mM}$ Mes ( $\mathrm{pH}$ 6.5), $0.5 \mathrm{mM} \mathrm{MgCl}_{2}$. The orientation of $\mathrm{L} 11$ on the GAC is further characterized by smallangle neutron scattering. The authors discuss the implications of a flexible $\mathrm{N}$-terminal domain for ribosomal co-factor interactions.

$\bullet$ 10. Jonker HR, Ilin S, Grimm SK, Wohnert J, Schwalbe H. L11 domain rearrangement upon binding to RNA and thiostrepton studied by NMR spectroscopy. Nucleic Acids Res 2007;35:441-454. [PubMed: 17169991]Structures of L11 and the GAC from Haloarcula marismortui 2JQ7 are examined here and the dynamics of the L11 protein are measured by ${ }^{15} \mathrm{~N}-\mathrm{NMR}$ relaxation methods in the free and bound form. Solutions contained $200 \mathrm{mM} \mathrm{KCl}, 20 \mathrm{mM}$ potassium phosphate, $\mathrm{pH}$ 6.1 .

11. Markus MA, Hinck AP, Huang S, Draper DE, Torchia DA. High resolution solution structure of ribosomal protein L11-C76, a helical protein with a flexible loop that becomes structured upon binding to RNA Nat. Struct Biol 1997;4:70-77.

12. Li W, Sengupta J, Rath BR, Frank J. Functional conformations of the L11-ribosomal RNA complex revealed by correlative analysis of cryo-EM and molecular dynamics simulations. RNA 2005;12:1240-1253. [PubMed: 16682558]

13. Wimberly BT, Guymon R, McCutcheon JP, White SW, Ramakrishnan V. A detailed view of a ribosomal active site: the structure of the L11-RNA complex. Cell 1999;97:491-502. [PubMed: 10338213]

• 14. Razga F, Koca J, Mokdad A, Sponer J. Elastic properties of ribosomal RNA building blocks: molecular dynamics of the GTPase-associated center rRNA. Nucleic Acids Res 2007;35:40074017. [PubMed: 17553840]The focus is on the hinge regions of the larger GAC RNA, but also investigates the motions of the site where L11 is bound. In my opinion, there are insufficient ions in the experiment to be able to make any conclusions about their role in stabilizing the RNA.

15. Ban N, Nissen P, Hansen J, Moore PB, Steitz TA. The complete atomic structure of the large ribosomal subunit at 2.4 resolution. Science 2000;289:905-920. [PubMed: 10937989] 
16. Sanbonmatsu KY, Joseph S, Tung CS. Simulating movement of tRNA into the ribosome during decoding. Proc Natl Acad Sci U S A 2005;102:15854-9. [PubMed: 16249344]

17. Perez A, Marchan I, Svozil D, Sponer J, Cheatham TE III, Laughton CA, Orozco M. Refinement of the AMBER force field for nucleic acids: improving the description of alpha/gamma conformers. Biophys J 2007;92:3817-3829. [PubMed: 17351000]A fix of ff99 DNA backbone torsions that may or may not be appropriate for RNA.

18. Mayaan E, Moser A, MacKerell AD Jr, York DM. CHARMM force field parameters for simulation of reactive intermediates in native and thio-substituted ribozymes. J Comput Chem 2007;28:495507. [PubMed: 17186477]

19. Dayie KT, Brodsky AS, Williamson JR. Base flexibility in HIV-2 TAR RNA mapped by solution ${ }^{15} \mathrm{~N},{ }^{13} \mathrm{C}$ NMR relaxation. J Mol Biol 2022;317:263-278. [PubMed: 11902842]

20. Sun X, Zhang Q, Al-Hashimi HM. Resolving fast and slow motions in the internal loop containing stem-loop 1 of HIV-1 that are modulated by $\mathrm{Mg}^{2+}$ binding: role in the kissing-duplex structural transition. Nucleic Acids Res 2007;35:1698-1713. [PubMed: 17311812]

21. Zhang Q, Sun X, Webb ED, Al-Hashimi HM. Resolving the motional modes that code for RNA adaptation. Science 2006;311:653-656. [PubMed: 16456078]

22. Zhang Q, Stelzer AC, Fisher CK, Al-Hashimi HM. Visualizing spatially correlated dynamics that directs RNA conformational transitions. Nature 2007;450:1263-1267. [PubMed: 18097416]

-23. Hansen AL, Al-Hashimi HM. Dynamics of large elongated RNA by NMR carbon relaxation. JACS 2007;129:16072-16082. Their elongated HIV-1 TAR RNA is examined here both free bound to argininamide (a Tat mimic). Binding of the small ligand alters the mobility of the bulged nucleotides at the junction of the two helices.

- 24. Musselman C, Al-Hashimi HM, Andriciocaei I. iRED analysis of TAR RNA reveals motional coupling, long-range correlations, and a dynamical hinge. Biophys J 2007;93:411-22. [PubMed: 17449677]A companion to the NMR work on the dynamics of the HIV-1 TAR RNA. iRED is used here to demonstrate that indeed the overall tumbling of the RNA is separable from the local motions, ensuring accurate experimental NMR measurements of dynamics.

25. Showalter SA, Baker NA, Tang C, Hall KB. Iron responsive element RNA flexibility described by NMR and isotropic reorientational eigenmode dynamics. J Biomol NMR 2005;32:179-193. [PubMed: 16132819]

26. Addess KJ, Basilion JP, Klausner RD, Rouault TA, Pardi A. Structure and dynamics of the iron responsive element RNA: implications for binding of the RNA by iron regulatory binding proteins. J Mol Biol 1997;274:72-83. [PubMed: 9398517]

27. Conn GL, Gittis AG, Lattman EE, Misra VK, Draper DE. A compact RNA tertiary structure contains a buried backbone-K+ complex. J Mol Biol 2002;318:963-973. [PubMed: 12054794]

• 28. Kavran JM, Steitz TA. Structure of the Base of the L7/L12 Stalk of the Haloarcula marismortui Large Ribosomal Subunit: Analysis of L11 Movements. J Mol Biol 2007;371:1047-1059. [PubMed: 17599351]

29. Humphrey W, Dalke A, Schulten K. VMD - Visual Molecular Dynamics. J Molec Graphics 1996;14:33-38. 


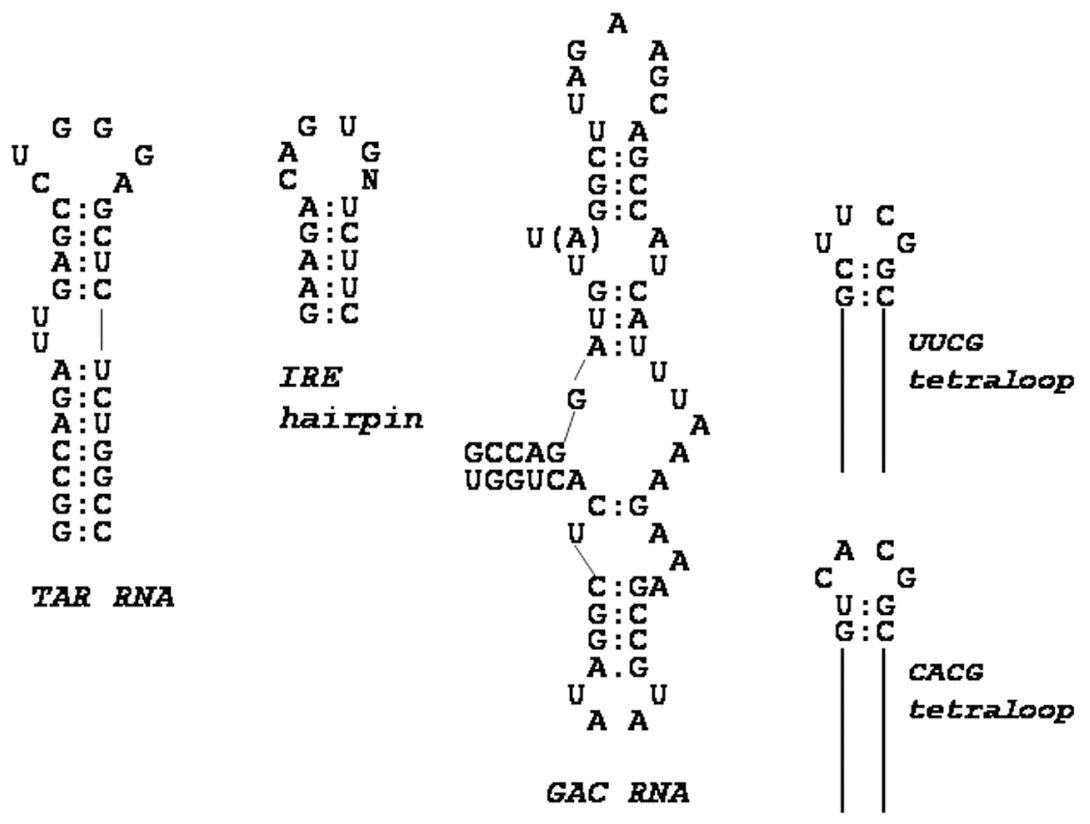

Figure 1.

Dynamic RNAs. The secondary structures of those RNAs for which both in vitro and in silico dynamics data are available. TAR RNA: NMR ref 20-24; MD: ref 25. IRE RNA hairpin: NMR ref 26,27; MD: 26. L11/GTPase Associated Center RNA. The upper stem is Helix 43, and the bottom stem is Helix 44. MD ref 12,14. UUCG/CAGC tetraloops: NMR/MD ref 2; MD 5,7. TAR is Transactivation response element; IRE is Iron Response Element. 


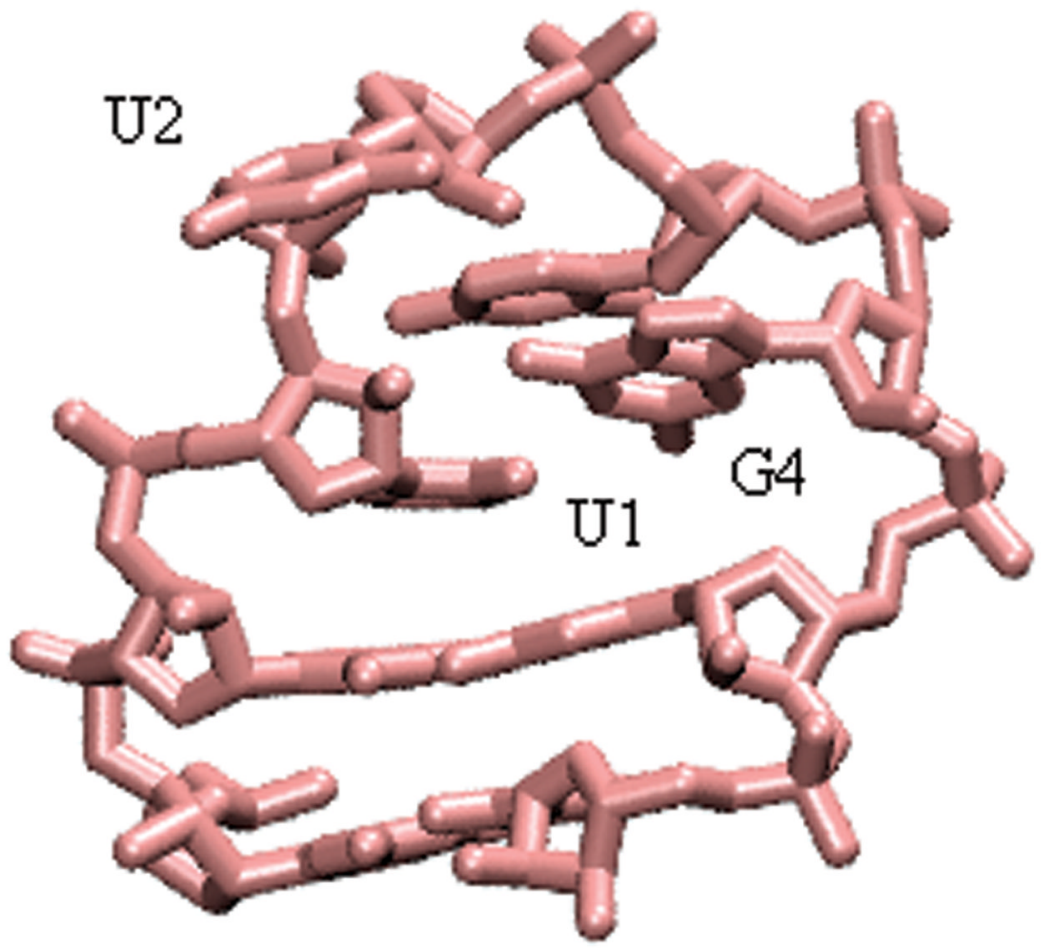

Figure 2.

The canonical UUCG loop structure. A tertiary structure of the $\mathrm{U}_{1} \mathrm{U}_{2} \mathrm{C}_{3} \mathrm{G}_{4}$ tetraloop. The $\mathrm{U}_{1}: \mathrm{G}_{4}$ bases are hydrogen bonding, $\mathrm{U}_{2}$ extends into solution with no interactions, and $\mathrm{C}_{3}$ stacks over the U:G base interaction. Image made with VMD (29). 


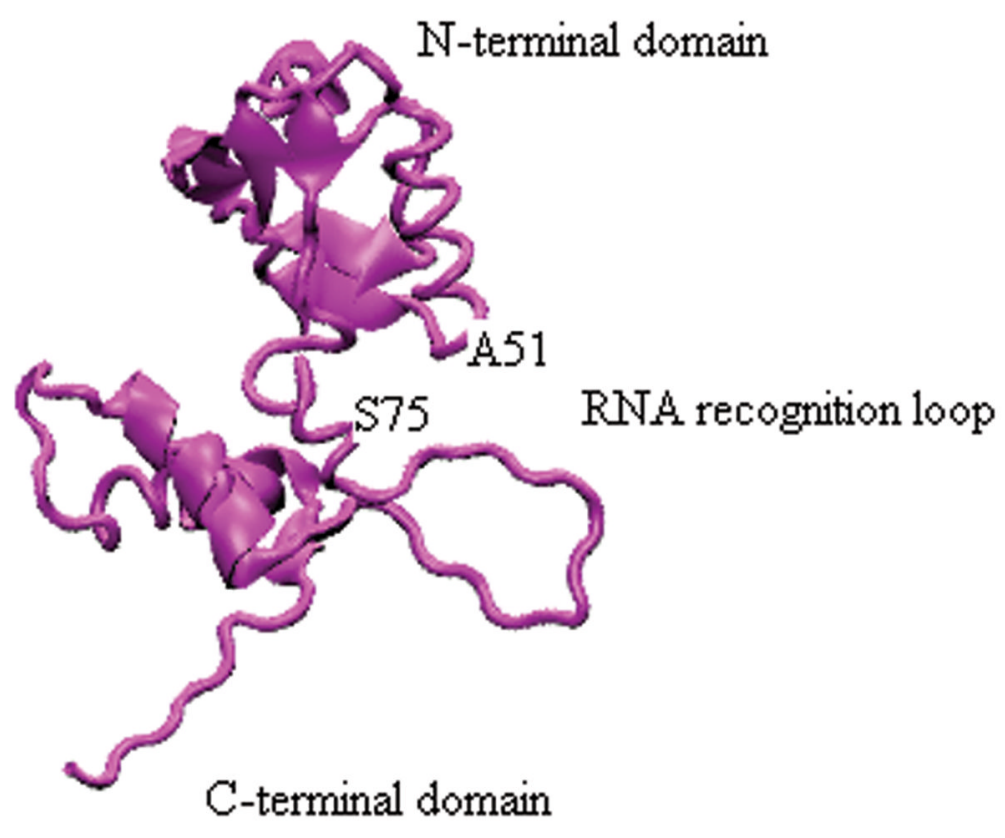

Figure 3.

An NMR structure of the free $B$ stearothermophilus L11 protein. Those regions of the protein that have significant dynamical motions (ps-ns) in the free form are labeled. Backbone dynamics of the protein were measured by NMR relaxation measurements (9). Image made with VMD (29). 

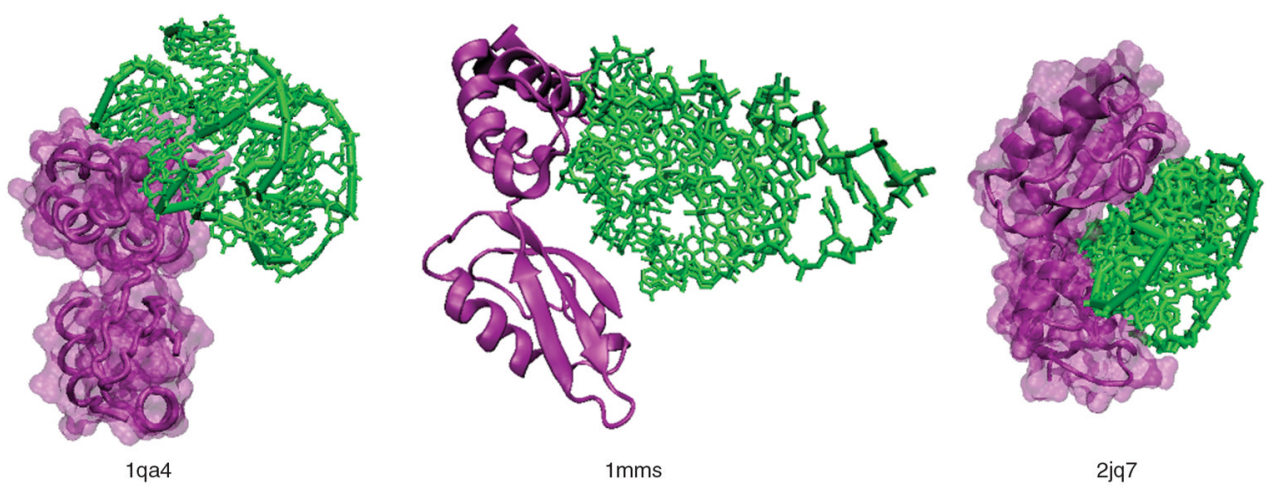

Figure 4.

Structures of the L11:GAC complex. Different orientations of the N-terminal domain of L11 protein in the complex with GAC RNA seen in NMR and crystal structures. NMR structure: pdb 2jq7 (9). X-ray crystal structure of the L11/CAG complex: pdb 1mms (13). X-ray crystal structure of the ribosome: pdb 1qa4 (28). Image made with VMD (29). 


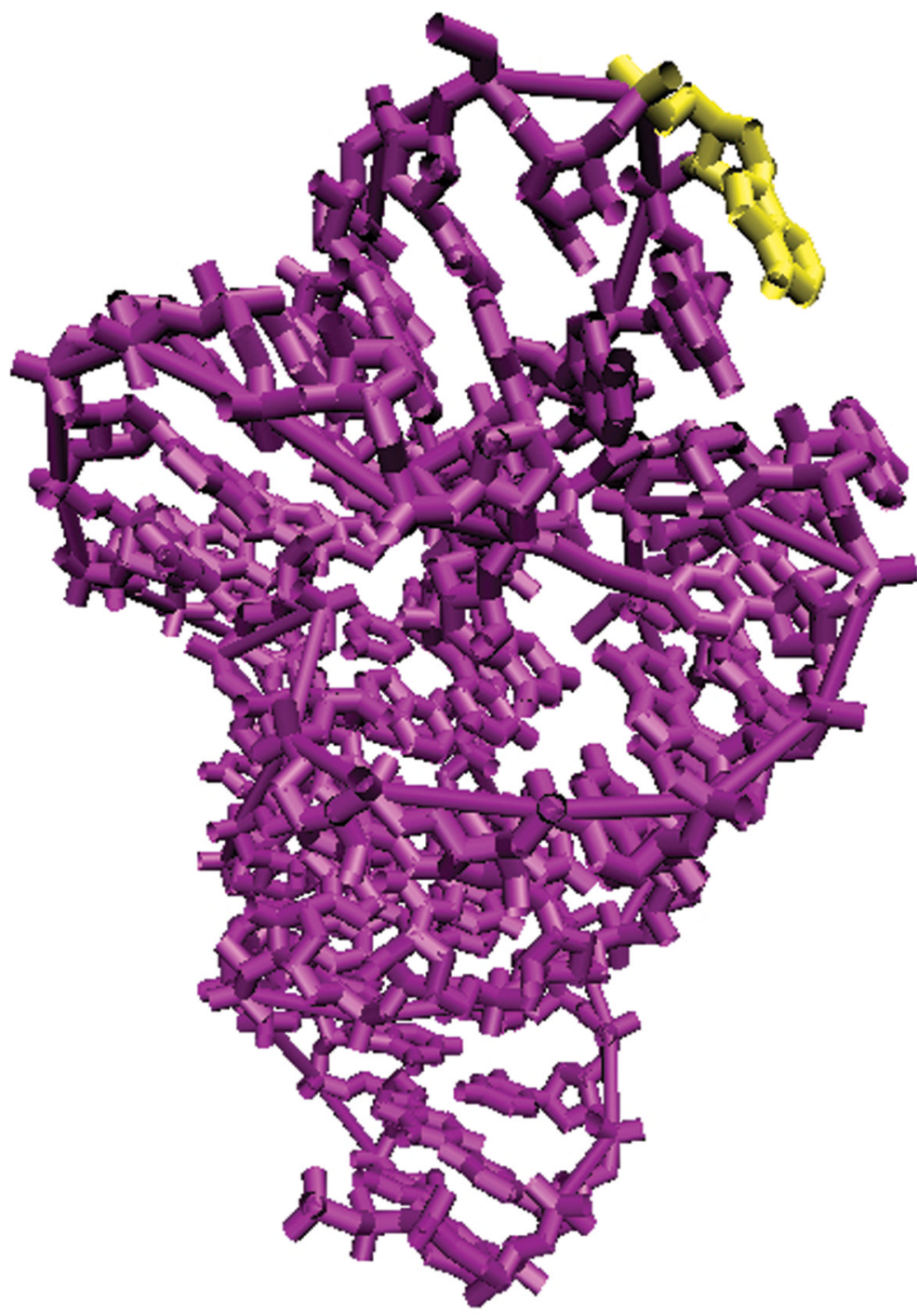

Figure 5.

The tertiary fold of a GAC. This crystal structure of the $E$ coli GAC was taken from a structure of the L11:GAC complex (27). The flexible A1067 is shown in yellow. A1067 is one of a very few nucleotides that shows dynamic motions in simulations. Image made with VMD (29). 